\title{
Extending the Limits of Resection for Colorectal Liver Metastases
}

\author{
Ernst $\operatorname{Klar}^{1}$ (B)
}

Received: 22 July 2016 / Accepted: 10 August 2016/Published online: 12 September 2016

(C) 2016 The Society for Surgery of the Alimentary Tract

Keywords Liver surgery $\cdot$ Colorectal metastases $\cdot$ Extended resection $\cdot$ ALPPS $\cdot$ Review

This year's international HPBA joint symposium continues a young series of events representing the SSAT outreach to international societies. The European-African-Hepato-Pancreatic-Biliary Association (E-AHPBA) reflected the goal to establish a mutual exchange between the SSAT and a subspecialized

This paper was originally presented as part of the SSAT/AHPBA/EAHPBA Joint Symposium: Extending the Limits of Resection for Colorectal Liver Metastases at the SSAT 57th annual meeting, May 2016, in San Diego, California. The other articles presented in the presentation are the following:

Extending the Limits of Resection for Colorectal Liver Metastases ENHANCED ONE STAGE SURGERY

Guido Torzilli, MD, PhD, FACS, Matteo Maria Cimino, MD

Extending the Limits of Resection for Colorectal Liver Metastases ALPPS for colorectal liver metastases

Prof. Dr. Hauke Lang

Extending the Limits of Resection for Colorectal Liver Metastases

Bilateral Disease

Thomas A. Aloia

Extending the Limits of Resection for Colorectal Liver Metastases

Positive Resection Margin and Outcome After Resection of Colorectal

Cancer Liver Metastases

Motaz Qadan

Michael I. D’Angelica

Ernst Klar

ernst.klar@med.uni-rostock.de

1 SSAT International Relations Committee, Rostock, Germany international society. In 2015, this project started with an outstanding SSAT/E-AHPBA joint session on "Hot Topics in Pancreatic Surgery" at the Manchester Surgical Week 2015 in Manchester, England. Both societies decided to continue having joint sessions in a mutual fashion at the respective annual meetings. At the Digestive Disease Week 2016 in San Diego, an extraordinary panel of international experts was set up focusing on "Extending the Limits of Resection for Colorectal Liver Metastases." The high-standing presentations were followed by an intense discussion of the different concepts to approach extended liver metastases by surgery. Presenting his series of one-stage hepatectomy, Dr. Guido Torzilli, Milan, Italy, showed impressive pictures of how to resect multiple diffuse metastases especially at deep locations. One key step consists of accurate flow analysis of communicating hepatic veins. Dr. Thomas Aloia, Houston, gave an impression of a tailored approach of one-stage, two-stage hepatectomy, and a combination of resection and ablation techniques. Dr. Michael D'Angelica, New York, emphasized a correlation between positive resection margin, disseminated or distant recurrences, and aggressive tumor biology. ALPPS was reevaluated by Dr. Hauke Lang, Mainz, Germany, who made clear that it should be reserved as an ultimate surgical option. As a new technical aspect, he showed that the patient might benefit even from a superficial transsection of the parenchyma inducing growth factor stimulation.

To offer more detailed information about this state-of-theart analysis, four short manuscripts reflecting this outstanding HPB-symposium are presented here. 